\title{
On Singular Cut-and-Pastes in the 3-Space with Applications to Link Theory
}

\author{
Fujitsugu HOSOKAWA and Shin'ichi SUZUKI
}

\begin{abstract}
In the study of surfaces in 3-manifolds, the so-called "cutand-paste" of surfaces is frequently used. In this paper, we generalize this method, in a sense, to singular-surfaces, and as an application, we prove that two collections of singular-disks in the 3 -space $R^{3}$ which span the same trivial link are link-homotopic in the upper-half 4 -space $R^{3}[0, \infty)$ keeping the link fixed.
\end{abstract}

Throughout the paper, we work in the piecewise linear category, consisting of simplicial complexes and piecewise linear maps.

\section{SINGULAR LOOPS IN A 2-CELL}

We denote by $\partial X$ and ${ }^{\circ} X$, respectively, the boundary and the interior of a manifold $X$. For a subcomplex $P$ in a complex $M$, by $N(P ; M)$ we denote a regular neighborhood of $P$ in $\cdot M$, that is, we construct the second derived of $M$ and take the closed star of $P$, see [H], [RS].

1991 Mathematics Subject Classification: 57M25, 55P99

Servicio publicaciones Univ. Complutense. Madrid, 1995. 
We shall say that a submanifold $X$ of a manifold $Y$ is proper iff $X \cap \partial Y=\partial X$.

By $R^{n}, D^{n}$ and $S^{n-1}$ we shall denote the Euclidean $n$-space, the standard $n$-cell and the standard $(n-1)$-sphere $\partial D^{n}$, respectively.

1.1. Definition. (1) Let $f: D^{1} \rightarrow M$ and $g: S^{1} \rightarrow M$ be non-degenerate continuous maps into a manifold $M$. Then, the images $f\left(D^{1}\right)=A$ and $g\left(S^{1}\right)=J$ will be called a singular-arc (or simply an arc) and a singular-loop (or simply a loop), respectively. In particular, $A$ and $J$ will be called a simple arc and a simple loop, respectively, if $f$ and $g$ are embeddings. The boundary of an arc $f\left(D^{1}\right)=A$ is the image $f\left(\partial D^{1}\right)$ of the boundary $\partial D^{1}$, and we denote it by $\partial^{*} A$.

(2) An arc $A^{-}$in a manifold $M$ is said to be proper iff $A \cap \partial F=\partial^{*} A$. $A$ loop $J$ in a manifold $M$ is said to be proper iff $J \subset{ }^{\circ} F$.

(3) Let $\mathcal{B}=B_{1} \cup \cdots \cup B_{n}$ be a finite union of proper arcs and proper loops in a 2-manifold $F^{2}$. A point $p$ in $\mathcal{B}$ is said to be a singular-point of multiplicity $k$, iff the number of the preimages of $p$ is $k$ with $k \geq 2$.

We shall say that $\mathcal{B}$ is normal, iff and

(i) $B$ has only a finite number of singular-points of multiplicity 2 ,

(ii) at every singular point of $\mathcal{B}, \mathcal{B}$ crosses transversally.

1.2. Lemma. Let $\mathcal{J}_{1}=J_{11} \cup \cdots \cup J_{1 m(1)}$ and $\mathcal{J}_{2}=J_{21} \cup \cdots \cup J_{2 m(2)}$ be finite unions of proper loops in a simply connected 2-manifold $F^{2}$ such that $\mathcal{J}_{1} \cap \mathcal{J}_{2}=\emptyset$. Then, there exists $j \in\{1, \cdots, m(1)\}$ or $k \in$ $\{1, \cdots, m(2)\}$ so that $J_{1 j}$ is contractible in $F^{2}-\mathcal{J}_{2}$ or $J_{2 k}$ is contractible in $F^{2}-\mathcal{J}_{1}$.

Proof. We may assume that $\mathcal{J}_{1} \cup \mathcal{J}_{2}$ is polygonal and normal.

Let $\mathcal{R}=\left\{R_{1}, \cdots, R_{r}\right\}$ be the set of regions of $F^{2}-{ }^{o} N\left(\mathcal{J}_{1} ; F^{2}\right)$. It will be noticed that $R_{1} \cup \cdots \cup R_{r} \supset \mathcal{J}_{2}$.

If there exist a loop, say $J_{2 k}$, of $\mathcal{J}_{2}$, and a simply connected region, say $R_{h}$, of $\mathcal{R}$ with $J_{2 k} \subset R_{h}$, then $J_{2 k}$ is contractible in $R_{h} \subset F^{2}-\mathcal{J}_{1}$, and so the proof is complete. 
So, we may assume that there exist some non-simply connected regions, say $Q_{1}, \cdots, Q_{q}$, of $\mathcal{R}$, so that $Q_{1} \cup \cdots \cup Q_{q} \supset \mathcal{J}_{2}$. Let $C_{1} \cup \cdots \cup$ $C_{s}=\partial Q_{1} \cup \cdots \cup \partial Q_{q}$ be the disjoint union of simple loops on $F^{2}$, and let $\Delta_{h}$ be the 2-cell on $F^{2}$ with $\partial \Delta_{h}=C_{h}(h=1, \cdots, s)$. We choose an innermost 2-cell, say $\Delta_{1}$, in $\left\{\Delta_{1}, \cdots, \Delta_{n}\right\}$, i.e. there is no other $\Delta_{h}$ in $\triangle_{1}$. Since $\triangle_{1}$ is not belong to $\mathcal{R}$ and $C_{1}=\partial \triangle_{1}$ is the one of the boundary curves $\partial Q_{1} \cup \cdots \cup \partial Q_{q}$, it holds that $\Delta_{1} \cap \mathcal{J}_{1} \neq \emptyset$, and since $\triangle_{1}$ does not contain any $Q_{1}, \cdots, Q_{q}$ and $\mathcal{J}_{2} \subset Q_{1} \cup \cdots \cup Q_{q}$, it holds that $\triangle_{1} \cap \mathcal{J}_{2}=\emptyset$. Now, any $J_{1 j}$ of $\mathcal{J}_{1}$ with $J_{1 j} \cap \triangle_{1} \neq \emptyset$ is contractible in $\triangle_{1} \subset F^{2}-\mathcal{J}_{2}$, and so the proof is complete.

In the same way as that Lemma 1.2. we have the following:

1.3. Theorem. Let $\mathcal{J}_{i}=J_{1} \cup \cdots \cup J_{\text {im(i) }}$ be a finite union of proper loops in a simply connected 2-manifold $F^{2}$ for $i=1, \cdots, \mu$, such that $\mathcal{J}_{i} \cap \mathcal{J}_{h}=\emptyset$ for $i \neq h$. Then, there exist $j \in\{1, \cdots, \mu\}$ and $k \in\{1, \cdots, m(j)\}$ so that $J_{j k}$ is contractible in $F^{2}-\bigcup_{i \neq j} \mathcal{J}_{i}$.

Proof. We prove this by induction on the number $\mu$ of the classes $\mathcal{J}_{i}$. The case of $\mu=1$ is trivial, and the case $\mu=2$ is Lemma 1.2. So, we assume that $\mu \geq 3$ and Theorem is true for $\mu-1$. We may assume that every $\mathcal{J}_{i}$ is polygonal and normal.

Let $\mathcal{R}=\left\{R_{1}, \cdots, R_{\tau}\right\}$ be the set of regions of $F^{2}-{ }^{o} N\left(\mathcal{J}_{1} ; F^{2}\right)$. It will be noted that $R_{1} \cup \cdots \cup R_{r} \supset \mathcal{J}_{2} \cup \cdots \cup \mathcal{J}_{\mu}$.

If there exist a loop, say $J_{j k}$, of $\mathcal{J}_{j}$ and a simply connected region, say $R_{h}$, of $\mathcal{R}$ with $J_{j k} \subset R_{h}$, then $\mathcal{J}_{i}^{\prime}=\mathcal{J}_{i} \cap R_{h}(i=2, \cdots, \mu)$ is a finite union of loops in the simply connected region $R_{h}$ satisfying the conditions of Theorem. By induction hypothesis, we have a loop, say $J_{j k}$, of $\mathcal{J}_{j}^{\prime} \subset \mathcal{J}_{j}$ so that $J_{j k}$ is contractible in $R_{h}-\bigcup_{i \neq 1, j} \mathcal{J}_{i}^{\prime} \subset F^{2}-\bigcup_{i \neq j} \mathcal{J}_{i}$, and so the proof is complete.

So, we may assume that there exist some non-simply connected regions, say $Q_{1}, \cdots, Q_{q}$ of $\mathcal{R}$, so that $Q_{1} \cup \cdots \cup Q_{q} \supset \mathcal{J}_{2} \cup \cdots \cup \mathcal{J}_{\mu}$. Now, the proof of this case, which is omitted here, is the same as that of Lemma 1.2.

In general, we have the following: 
1.4. Theorem. Let $\mathcal{A}_{i}=A_{i 1} \cup \cdots \cup A_{i n(i)}$ be a finite union of proper arcs in a simply connected 2-manifold $F^{2}$ for $i=1, \cdots, \mu$, and let $\mathcal{J}_{i}=J_{i 1} \cup \cdots \cup J_{i m(i)}$ be a finite union of proper loops in $F^{2}$, such that $\left(\mathcal{A}_{i} \cup \mathcal{J}_{i}\right) \cap\left(\mathcal{A}_{h} \cup \mathcal{J}_{h}\right)=\emptyset$ for $i \neq h$. Then, there exist $j \in\{1, \cdots, \mu\}$ and $k \in\{1, \cdots, m(j)\}$ so that $J_{j k}$ is contractible in $F^{2}-\bigcup_{i \neq j}\left(\mathcal{A}_{i} \cup \mathcal{J}_{i}\right)$.

Proof. We may assume that every $\mathcal{A}_{i} \cup \mathcal{J}_{i}$ is polygonal and normal.

Since every region of $F^{2}-{ }^{o} N\left(\mathcal{A}_{i} ; F^{2}\right)$ is simply connected, the proof of Theorem is similar to that of Theorem 1.3, and so it is omitted here.

\section{SINGULAR SPHERES IN A 3-CELL}

In this section, we will discuss singular 2-spheres in a 3-cell and prove similar theorems to those in the previous section.

First let us explain several well-known facts to be used in the sequel.

If a compact 3-manifold $M$ is embeddable in the 3-sphere $S^{3}$, then there is a 1-complex $G$ in $S^{3}$ such that the exterior $S^{3}-{ }^{0} N\left(G ; S^{3}\right)$ is homeomorphic to $M$ by Fox [F].

A 1-complex $G$ in $S^{3}$ is said to be split, iff there exists a 2-sphere $S \subset S^{3}-G$, such that both components of $S^{3}-S$ contain points of $G$. If a 1-complex $G \subset S^{3}$ is not split, then the exterior $S^{3}-{ }^{\circ} N\left(G ; S^{3}\right)$ is aspherical, i.e. the second homotopy group $\pi_{2}\left(S^{3}-{ }^{\circ} N\left(G ; S^{3}\right)\right)=\{0\}$, by Papakyriakopoulos [P]. In particular, if $G \subset S^{3}$ is a connected 1complex, then $S^{3}-{ }^{o} N\left(G ; S^{3}\right)$ is aspherical.

We will call a compact 3-manifold $M$ an aspherical region, iff $M$ is embeddable in $S^{3}$ and aspherical.

It holds the following:

2.1. Proposition. (i) If a compact 3-manifold $M$ is embeddable in $S^{3}$ and $\partial M$ is connected, then $M$ is an aspherical region.

(2) Let $M$ be an aspherical region with connected boundary $\partial M$ and let $F \subset{ }^{\circ} M$ be a closed connected 2-manifold. Then, there exists an aspherical region $R$ in $M$ with $\partial R=F$.

The following corresponds to Definition 1.1. 
2.2. Definition. (1) Let $f: F^{2} \rightarrow M$ be a non-degenerate continuous map of a compact 2-manifold $F^{2}$ into a manifold $M$. Then, the image $f\left(F^{2}\right)=F$ will be called a singular-surface. In particular, singular-surfaces $f\left(D^{2}\right)=D$ and $g\left(S^{2}\right)=S$ will be called a singulardisk and singular-sphere, respectively.

The boundary of a singular-surface $f\left(F^{2}\right)=F$ is the image $f\left(\partial F^{2}\right)$, and we denote it by $\partial^{*} F$.

(2) A singular-surface $F$ in a manifold $M$ is said to be proper iff $F \cap \partial M=\partial^{*} F$.

(3) Let $F$ be a proper singular-surface in a 3-manifold $M$. A point $p$ in $F$ is a singular-point of multiplicity $k$, iff the number of the preimages of $p$ is $k$ with $k \geq 2$.

We shall say that $F$ is normal, iff

(i) F has only singular-points of multiplicity 2 and 3,

(ii) the set of singular-points of multiplicity 2 is a finite number of polygonal curves, that is, singular-arcs and singular-loops, which will be called double-lines,

(iii) the set of singular-points of multiplicity 3 consists of a finite number of points which are intersection points of the double-lines, which will be called triple-points, and

(iv) at every singular-point of multiplicity $2, F$ crosses transversally.

In fact, every singular-point $p$ of $F$ has one of the neighborhood described in Figure 1, and it is well known that every singular-surface may be $\varepsilon$-approximated by such a normal one. 

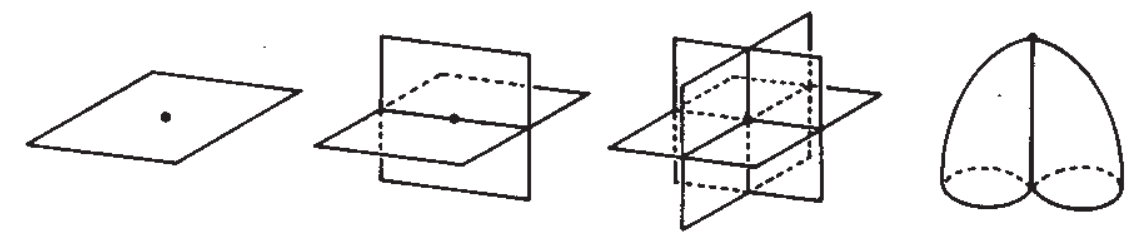

regular point double point triple point branch point

\section{Figure 1}

2.3. Lemma. Let $S_{1}=S_{11} \cup \cdots \cup S_{1 m(1)}$ and $S_{2}=S_{21} \cup \cdots \cup S_{2 m(2)}$ be finite unions of proper singular-spheres in an aspherical region $M$ with connected boundary $\partial M$ such that $\mathcal{S}_{1} \cap \mathcal{S}_{2}=\emptyset$. Then, there exists $j \in\{1, \cdots, m(1)\}$ or $k \in\{1, \cdots, m(2)\}$ so that $S_{1 j}$ is contractible in $M-\mathcal{S}_{2}$ or $S_{2 k}$ is contractible in $M-S_{1}$.

Proof. We may assume that $\mathcal{S}_{1} \cup \mathcal{S}_{2}$ is normal. The proof of this Lemma is similar to that of Lemma 1.2.

Let $\mathcal{R}=\left\{R_{1}, \cdots, R_{r}\right\}$ be the set of regions of $M-{ }^{o} N\left(\mathcal{S}_{1} ; M\right)$. It will be noted that $R_{1} \cup \cdots \cup R_{r} \supset S_{2}$.

If there exist a singular-sphere, say $S_{2 k}$, of $\mathcal{S}_{2}$ and an aspherical region, say $R_{h}$, of $\mathcal{R}$ with $S_{2 k} \subset R_{h}$, then $S_{2 k}$ is contractible in $R_{h} \subset$ $M-S_{1}$, and we are finished.

So, we may assume that there exist some spherical regions, say $Q_{1}, \cdots, Q_{q}$, in $\mathcal{R}$, so that $Q_{1} \cup \cdots \cup Q_{q} \supset \mathcal{S}_{2}$. Let $F_{1} \cup \cdots \cup F_{s}=$ $\partial Q_{1} \cup \cdots \cup \partial Q_{q}$ be the disjoint union of closed connected 2-manifolds, and let $M_{h}$ be the aspherical region in $M$ with $\partial M_{h}=F_{h}$ for $h=1, \cdots, s$, see Proposition 2.1 (2). We choose an innermost region, say $M_{1}$, in these aspherical regions, that is, there are no other $M_{h}$ in $M_{1}$. Then, by the same way as the proof of Lemma 1.2 , it is easily checked that $M_{1} \cap \mathcal{S}_{1} \neq \emptyset$ and $M_{1} \cap \mathcal{S}_{2}=\emptyset$. Now, any $S_{1 j}$ of $\mathcal{S}_{1}$ with $S_{1 j} \cap M_{1} \neq \emptyset$ is contractible in $M_{1} \subset M-S_{2}$, and completing the proof. 
The following theorems correspond to Theorems 1.3 and 1.4, respectively.

2.4. Theorem. Let $\mathcal{S}_{i}=S_{i 1} \cup \cdots \cup S_{i m(i)}$ be a finite union of proper singular-spheres in an aspherical region $M$ with connected boundary $\partial M$ for $i=1, \cdots, \mu$, such that $\mathcal{S}_{i} \cap \mathcal{S}_{h}=\emptyset$ for $i \neq h$. Then, there exist $j \in$ $\{1, \cdots, \mu\}$ and $k \in\{1, \cdots, m(j)\}$ so that $S_{j k}$ is contractible in $M-\bigcup_{i \neq j} \mathcal{S}_{i}$.

Proof. The proof is similar to that of Lemma 2.3, and is word for word that of Theorem 1.3.

2.5. Theorem. Let $M$ be an aspherical region with connected boundary $\partial M$. Let $\mathcal{D}_{i}=D_{i 1} \cup \cdots \cup D_{\text {in(i) }}$ and $\mathcal{S}_{i}=S_{i 1} \cup \cdots \cup S_{i m(i)}$ be finite unions of proper singular-disks and proper singular-spheres in $M$, respectively, for $i=1, \cdots, \mu$, such that $\left(\mathcal{D}_{i} \cup \mathcal{S}_{i}\right) \cap\left(\mathcal{D}_{h} \cup \mathcal{S}_{h}\right)=\emptyset$ for $i \neq h$. Then, there exist $j \in\{1, \cdots, \mu\}$ and $k \in\{1, \cdots, m(j)\}$, so that $S_{j k}$ is contractible in $M-\bigcup_{i \neq j}\left(\mathcal{D}_{i} \cup \mathcal{S}_{i}\right)$.

Proof. We may assume that every $\mathcal{D}_{i} \cup \mathcal{S}_{i}$ is normal. Since every region $R$ of $M-{ }^{\circ} N\left(\mathcal{D}_{i} ; M\right)$ is an aspherical region, the proof of this Theorem is similar to that of Theorem 2.4, and is word for word that of Theorem 1.4.

\section{SINGULAR CUT-AND-PASTES}

3.1. Definition. Let $M^{3}$ be a 3-manifold, and let $E^{2}$ be a compact 2-manifold in ${ }^{\circ} M^{3}$. Let $f: F^{2} \rightarrow M^{3}$ be a non-degenerate continuous map of a compact 2-manifold $F^{2}$ into $M^{3}$ such that

(i) $f\left(F^{2}\right)=F$ is a normal singular-surface,

(ii) $F$ intersects with $E^{2}$ transversally, and

(iii) no triple-point and no branch point of $F$ lie on $E^{2}$.

Then, the intersection $F \cap E^{2}$ consists of a finite number of arcs and loops. Let $J$ be a loop in $F \cap E^{2}$, and let $J^{*}$ be the preimage of $J$ in $F^{2}: J^{*}$ is a simple loop. We suppose that $J^{*}$ is 2-sided on $F^{2}$, and let $F^{2}$ be the 2-manifold obtained from $F^{2}$ by attaching a 2-handle along $J^{*}$. In fact, we define $F^{\prime 2}$ as follows: We take a homeomorphism 
$h^{2}: \partial D^{2} \times D^{1} \rightarrow N\left(J^{*} ; F^{2}\right)$ with $h^{2}\left(\partial D^{2} \times 0\right)=J^{*}$, and let $F^{2}=$ $\left(F^{2}-{ }^{\circ} N\left(J^{*} ; F^{2}\right)\right) \cup h^{2}\left(D^{2} \times \partial D^{1}\right)$.

Now, we suppose that $J$ is contractible on $E^{2}$. Then, we have a non-degenerate continuous map, say $g$, of $D^{2}$ into $E^{2} \subset M^{3}$ such that $g\left(\partial D^{2}\right)=J$. Using the product structure $N\left(E^{2} ; M^{3}\right) \cong E^{2} \times D^{1}$, we define a non-degenerate continuous map $f^{\prime}: F^{\prime 2} \rightarrow M^{3}$ as follows:

$$
\begin{aligned}
f^{\prime} \mid F^{\prime 2}-h^{2}\left(D^{2} \times \partial D^{1}\right) & =f \mid F^{2}-h^{2}\left(\partial D^{2} \times D^{1}\right), \\
f^{\prime}\left(h^{2}\left(D^{2} \times \partial D^{1}\right)\right) & =g\left(D^{2}\right) \times \partial D^{1} \subset E^{2} \times D^{1} .
\end{aligned}
$$

We say that $F^{\prime}=f^{\prime}\left(F^{\prime 2}\right)$ is obtained from $F=f\left(F^{2}\right)$ by a cutand-paste along $J \subset E^{2}$, and we denote simply by $F \rightarrow F^{\prime}$.

It will be noticed that $F^{\prime} \cap E^{2}=F \cap E^{2}-J$ and that $F^{\prime 2}=D^{2} \amalg S^{2}$ (a disjoint union) provided that $F^{2}=D^{2}$ and $F^{\prime 2}=S^{2}$ II $S^{2}$ provided that $F^{2}=S^{2}$.

3.2. Theorem. Let $\mathcal{O}_{i}=O_{i 1} \cup \cdots \cup O_{i n(i)}$ be a trivial link in the 3-sphere $S^{3}$ (or the 3-space $R^{3}$ ) for $i=1, \cdots, \mu$, such that $\mathcal{O}_{1} \cup \cdots \cup \mathcal{O}_{\mu}$ is also a trivial link. Let $\mathcal{D}_{i}=D_{i 1} \cup \cdots \cup D_{i n(i)}$ be a finite union of normal singular-disks in $S^{3}$ for $i=1, \cdots, \mu$, such that $\partial^{*} D_{i j}=O_{i j}$ for $i=1, \cdots, \mu$ and $j=1, \cdots, n(i)$, and $\mathcal{D}_{i} \cap \mathcal{D}_{h}=$ for $i \neq h$.

Let $\mathcal{D}_{i}^{*}=D_{i 1}^{*} \cup \cdots \cup D_{i n(i)}^{*}$ be mutually disjoint 2-cells in $S^{3}$ (or $\left.R^{3}\right)$ for $i=1, \cdots, \mu$, such that $\partial D_{i j}^{*}=O_{i j}$ for $i=1, \cdots, \mu$ and $j=$ $1, \cdots, n(i)$, and $\mathcal{D}_{i}^{*} \cap \mathcal{D}_{h}^{*}=\emptyset$ for $i \neq h$.

We suppose that $\mathcal{D}_{1} \cup \cdots \cup \mathcal{D}_{\mu}$ intersects with $\mathcal{D}_{1}^{*} \cup \cdots \cup \mathcal{D}_{\mu}^{*}$ transversally, and any triple-point and any branch-point of $\mathcal{D}_{1} \cup \cdots \cup \mathcal{D}_{\mu}$ do not lie on $\mathcal{D}_{1}^{*} \cup \cdots \cup \mathcal{D}_{\mu}^{*}$.

Then, there exists a finite sequence of cut-and-pastes

$$
\begin{gathered}
\mathcal{D}_{1} \cup \cdots \cup \mathcal{D}_{\mu}=\mathcal{D}_{1}^{(0)} \cup \cdots \cup \mathcal{D}_{\mu}^{(0)} \rightarrow \mathcal{D}_{1}^{(1)} \cup \cdots \cup \mathcal{D}_{\mu}^{(1)} \rightarrow \cdots \\
\rightarrow \mathcal{D}_{1}^{(u)} \cup \cdots \cup \mathcal{D}_{\mu}^{(u)} \rightarrow \cdots \rightarrow \mathcal{D}_{1}^{(w)} \cup \cdots \cup \mathcal{D}_{\mu}^{(w)} \\
\text { along }\left(\mathcal{D}_{1} \cup \cdots \cup \mathcal{D}_{\mu}\right) \cap\left(\mathcal{D}_{1}^{*} \cup \cdots \cup \mathcal{D}_{\mu}^{*}\right) \subset \mathcal{D}_{1}^{*} \cup \cdots \cup \mathcal{D}_{\mu}^{*} \text { such that }
\end{gathered}
$$


(1) $\mathcal{D}_{i}^{(u)}=D_{i 1}^{(u)} \cup \cdots \cup D_{i n(i)}^{(u)} \cup S_{i 1}^{(u)} \cup \cdots \cup S_{i m(i)}^{(u)}$, where $D_{i j}^{(u)}$ is a singular-disk with $\partial^{*} D_{i j}^{(u)}=O_{i j}$ and $S_{i s}^{(u)}$ is a singular-sphere, for $i=1, \cdots, \mu ; j=1, \cdots, n(i) ; u=1, \cdots, w ; s=1, \cdots, m(i)$,

(2) $\mathcal{D}_{i}^{(u)} \cap \mathcal{D}_{h}^{(u)}=\emptyset$ for $i \neq h, u=1, \cdots, w$, and

(3) $\mathcal{D}_{i}^{(w)} \cap \mathcal{D}_{h}^{*}=\emptyset$ for $i \neq h$, and $\mathcal{D}_{i}^{(w)} \cap \mathcal{D}_{i}^{*}=\left(D_{i 1}^{(w)} \cup \cdots \cup D_{i n(i)}^{(w)}\right) \cap \mathcal{D}_{i}^{*}$ consists of a finite number of proper arcs in $\mathcal{D}_{i}^{*}$.

Proof. From our hypothesis, $D_{i j} \cap D_{h k}^{*}$ consists of proper loops in $D_{h k}^{*}$ provided that $i \neq h$, and $D_{i j} \cap D_{i k}^{*}$ consists of proper loops and proper arcs in $D_{i k}^{*}$ for every $i, j, k$. Therefore, by the induction on the number $n=n(1)+\cdots+n(\mu)$ of 2 -cells in $\mathcal{D}_{1}^{*} \cup \cdots \cup \mathcal{D}_{\mu}^{*}$, it suffices to show that there exists a finite sequence of cut-and-pastes of $\mathcal{D}_{1} \cup \cdots \cup \mathcal{D}_{\mu}$ along proper loops $\left(\mathcal{D}_{1} \cup \cdots \cup \mathcal{D}_{\mu}\right) \cap D_{11}^{*} \subset D_{11}^{*}$ so that $\mathcal{D}_{1}^{(u)} \cup \cdots \cup \mathcal{D}_{\mu}^{(u)}$ satisfies the conditions (1), (2) and

(3) $\mathcal{D}_{i}^{(w)} \cap D_{11}^{*}=\emptyset$ and $\mathcal{D}_{i}^{(w)} \cap D_{1 j}^{*}=\mathcal{D}_{i} \cap D_{1 j}^{*}$ for $i=2, \cdots, t$ and $j=2, \cdots, n(1)$, and $\mathcal{D}_{1}^{(w)} \cap D_{11}^{*}$ consists of a finite number of proper arcs in $D_{11}^{*}$ and $\mathcal{D}_{1}^{(w)} \cap D_{1 j}^{*}=\mathcal{D}_{1} \cap D_{1 j}^{*}$ for $j=2, \cdots, n(1)$.

We consider $\mathcal{D}_{1} \cup \cdots \cup \mathcal{D}_{\mu}$ and $D_{11}^{*}$. Let $\mathcal{A}_{1}=A_{11} \cup \cdots \cup A_{1 a(1)}$ be the collection of proper arcs in $\mathcal{D}_{1} \cap D_{11}^{*}$ on $D_{11}^{*}$, and let $\mathcal{A}_{i}=\emptyset$ be the collection of proper arcs in $\mathcal{D}_{i} \cap D_{11}^{*}$ for $i=2, \cdots, \mu$. Let $\mathcal{J}_{i}=$ $J_{i 1} \cup \cdots \cup J_{i b(i)}$ be a collection of proper loops in $\mathcal{D}_{i} \cap D_{11}^{*}$ on $D_{11}^{*}$ for $i=1, \cdots, \mu$. Then, $\mathcal{A}_{i} \cup \mathcal{J}_{i}$ satisfies the assumptions in Theorem 1.4, and so there exists a loop $J_{j k}$ of some $\mathcal{J}_{j}$ such that $J_{j k}$ is contractible in $D_{11}^{*}-\bigcup_{i \neq j}\left(\mathcal{A}_{i} \cup \mathcal{J}_{i}\right)$. We have a non-degenerate continuous map $g: D^{2} \rightarrow$ $D_{11}^{*}$ such that $g\left(D^{2}\right) \cap\left(\mathcal{A}_{i} \cup \mathcal{J}_{i}\right)=\emptyset$ for $i \neq j$. Using this $g$, we perform the first cut-and-paste for $\mathcal{D}_{j} \subset \mathcal{D}_{1} \cup \cdots \cup \mathcal{D}_{\mu}=\mathcal{D}_{1}^{(0)} \cup \cdots \cup \mathcal{D}_{\mu}^{(0)}$ and obtain $\mathcal{D}_{1}^{(1)} \cup \cdots \cup \mathcal{D}_{\mu}^{(1)}$. Let $w$ be the number of loops in $\left(\mathcal{D}_{1} \cup \cdots \cup \mathcal{D}_{\mu}\right) \cap D_{11}^{*}$. By the repetition of the procedure $w$ times, we can get rid of all loops in $\left(\mathcal{D}_{1} \cup \cdots \cup \mathcal{D}_{\mu}\right) \cap D_{11}^{*}$, and it is easily checked that $\mathcal{D}_{1}^{(u)} \cup \cdots \cup \mathcal{D}_{\mu}^{(u)}$ satisfies the required conditions for $u=1, \cdots, w$, and we complete the proof of Theorem.

3.3. Remarks. (1) From the proof of Theorem 3.2, we know that $w$ is the number of loops in $\left(\mathcal{D}_{1} \cup \ldots \cup \mathcal{D}_{\mu}\right) \cap\left(\mathcal{D}_{1}^{*} \cup \ldots \cup \mathcal{D}_{\mu}^{*}\right)$ 
and $w=m(1)+\cdots+m(\mu)$, which is the number of singular-spheres in $\mathcal{D}_{1}^{(w)} \cup \cdots \cup \mathcal{D}_{\mu}^{(w)}$.

(2) Let $D$ and $D^{*}$ be a normal singular-disk and a 2-cell, respectively, in $S^{3}$ (or $R^{3}$ ) such that $\partial^{*} D=\partial D^{*}=O$ (a trivial knot). Let $A$ be a proper arc of $D \cap D^{*}$ in $D^{*}$ and let $\alpha$ be a simple arc in $O$ with $\partial \alpha=\partial^{*} A$. Since $A \cup \alpha$ is contractible in $D^{*}$, we can formulate a cut-and-paste of $D$ along $A \cup \alpha \subset D^{*}$ as the same way as Definition 3.1 except for obvious modifications, so that $D \rightarrow D^{\prime}=D_{1}^{\prime} \cup S_{1}^{\prime}$, where $S_{1}^{\prime}$ is a singular-sphere and $D_{1}^{\prime}$ is a singular-disk with $\partial^{*} D_{1}^{\prime}=O$.

Now, in the notation and assumptions of Theorem 3.2, we suppose that $D_{i j} \cap D_{i k}^{*}$ does not contain proper arcs on $D_{i k}^{*}$ for $i=1, \cdots, \mu$ and $j \neq k$. Then, we can remove proper arcs of $\mathcal{D}_{i}^{(w)} \cap \mathcal{D}_{i}^{*}$ by a finite sequence of the modified cut-and-pastes.

\section{APPLICATIONS TO LINK THEORY}

A continuous image of the 3 -cell $D^{3}$ will be called a singular-ball. The boundary of a singular-ball $B$ is the image of $\partial D^{3}$, and we denote it by $\partial^{*} B$.

We use here the same notation as that of Section 0 in [KSS].

The following is a generalization of Horibe-Yanagawa's Lemma [KSS, Lemma 1.6] in a sense.

4.1. Theorem. In the notation and assumptions of Theorem 3.2, let $\Sigma_{i}=\Sigma_{i 1} \cup \cdots \cup \Sigma_{i n(i)}$ be a finite union of singular-spheres in $R^{3}[0,1]$ defined by

$$
\Sigma_{i j}=D_{i j}[0] \cup O_{i j} \times[0,1] \cup D_{i j}^{*}[1]
$$

for $i=1, \cdots, \mu$ and $j=1, \cdots, n(i)$. Then, we can find a finite union of singular-balls $B_{i}=B_{i 1} \cup \cdots \cup B_{i n(i)}$ in $R^{3}[0, \infty)$ for $i=1, \cdots, \mu$, such that $\partial^{*} B_{i j}=\Sigma_{i j}$ for every $i$ and $j$, and $\mathcal{B}_{i} \cap \mathcal{B}_{h}=\emptyset$ for $i \neq h$.

Proof. The proof is similar to that of [KSS, Lemma 1.6]. We shall construct the required singular-balls $\mathcal{B}_{1} \cup \cdots \cup \mathcal{B}_{\mu}$ by specifying the cross-sections $B_{i j} \cap R^{3}[t]$ for all $i$ and $j$. 
Under the notation of Theorem 3.2, we also use Theorem 3.2. Let $g_{u}: D^{2} \rightarrow \mathcal{D}_{1}^{*} \cup \cdots \cup \mathcal{D}_{\mu}^{*}(u=1, \cdots, w)$ be a non-degenerate continuous map such that we perform the $u$-th cut-and-paste

$$
\mathcal{D}_{1}^{(u-1)} \cup \cdots \cup \mathcal{D}_{\mu}^{(u-1)} \rightarrow \mathcal{D}_{1}^{(u)} \cup \cdots \cup \mathcal{D}_{\mu}^{(u)}
$$

in Theorem 3.2 along the loop $g_{u}\left(\partial D^{2}\right)$ under $g_{u}$. We extend $g_{u}$ to a continuous map

$$
g_{u}^{\#}: h^{2}\left(D^{2} \times D^{1}\right) \rightarrow N\left(\mathcal{D}_{1}^{*} \cup \cdots \cup \mathcal{D}_{\mu}^{*} ; R^{3}\right) \cong\left(\mathcal{D}_{1}^{*} \cup \cdots \cup \mathcal{D}_{\mu}^{*}\right) \times D^{1}
$$

of the 3-cell $h^{2}\left(D^{2} \times D^{1}\right)$ naturally, and we denote the singular-ball $g_{u}^{\#}\left(h^{2}\left(D^{2} \times D^{1}\right)\right)$ by $H_{u}$. We divide the interval $[0,1]$ into the subintervals $\left[0, t_{1}\right],\left[t_{1}, t_{2}\right], \cdots,\left[t_{w-1}, t_{w}\right],\left[t_{w}, 1\right]$, where $t_{u}=u /(w+1), u=1, \cdots, w$. Let

$$
\begin{gathered}
\left(\mathcal{B}_{1} \cup \cdots \cup \mathcal{B}_{\mu}\right) \cap R^{3}[t]=\left(\mathcal{D}_{1} \cup \cdots \cup \mathcal{D}_{\mu}\right)[t] \text { for } 0 \leq t<t_{1} \\
\left(\mathcal{B}_{1} \cup \cdots \cup \mathcal{B}_{\mu}\right) \cap R^{3}\left[t_{1}\right]=\left(\mathcal{D}_{1} \cup \cdots \cup \mathcal{D}_{\mu} \cup H_{1}\right)\left[t_{1}\right] \\
\left(\mathcal{B}_{1} \cup \cdots \cup \mathcal{B}_{\mu}\right) \cap R^{3}[t]=\left(\mathcal{D}_{1}^{(1)} \cup \cdots \cup \mathcal{D}_{\mu}^{(1)}\right)[t] \text { for } t_{1}<t<t_{2}, \\
\cdots \cdots \cdots \\
\left(\mathcal{B}_{1} \cup \cdots \cup \mathcal{B}_{\mu}\right) \cap R^{3}[t]=\left(\mathcal{D}_{1}^{(u-1)} \cup \cdots \cup \mathcal{D}_{\mu}^{(u-1)}\right)[t] \text { for } t_{u-1}<t<t_{u}, \\
\left(\mathcal{B}_{1} \cup \cdots \cup \mathcal{B}_{\mu}\right) \cap R^{3}\left[t_{u}\right]=\left(\mathcal{D}_{1}^{(u-1)} \cup \cdots \cup \mathcal{D}_{\mu}^{(u-1)} \cup H_{u}\right)\left[t_{u}\right] \\
\left(B_{1} \cup \cdots \cup \mathcal{B}_{\mu}\right) \cap R^{3}[t]=\left(\mathcal{D}_{1}^{(u)} \cup \cdots \cup \mathcal{D}_{\mu}^{(u)}\right)[t] \text { for } t_{u}<t<t_{u+1}, \\
\quad \cdots \cdots \cdots \\
\left(\mathcal{B}_{1} \cup \cdots \cup \mathcal{B}_{\mu}\right) \cap R^{3}\left[t_{w}\right]=\left(\mathcal{D}_{1}^{(w-1)} \cup \cdots \cup \mathcal{D}_{\mu}^{(w-1)} \cup H_{w}\right)\left[t_{w}\right] \\
\left(\mathcal{B}_{1} \cup \cdots \cup \mathcal{B}_{\mu}\right) \cap R^{3}[t]=\left(\mathcal{D}_{1}^{(w)} \cup \cdots \cup \mathcal{D}_{\mu}^{(w)}\right)[t] \text { for } t_{w}<t \leq 1 .
\end{gathered}
$$

Thus, we constructed $\left(\mathcal{B}_{1} \cup \cdots \cup \mathcal{B}_{\mu}\right) \cap R^{3}[0,1]$ which consists of $n=n(1)+\cdots+n(\mu)$ singular-balls with $w=m(1)+\cdots+m(\mu)$ singularballs removed. 
Let $S_{i j}^{(w)}=D_{i j}^{(w)} \cup D_{i j}^{*}$ be the singular-sphere for $i=1, \cdots, \mu$ and $j=$ $m(i)+1, \ldots, m(i)+n(i)$, and let $S_{i}=\mathcal{D}_{i}^{(w)} \cup \mathcal{D}_{i}^{*}=S_{i 1}^{(w)} \cup \cdots \cup S_{i m(i)+n(i)}^{(w)}$, which consists of $m(i)+n(i)$ singular-spheres in $R^{3}$. From Theorem $3.2(2)$ and (3), it is easy to see that $\mathcal{S}_{i} \cap \mathcal{S}_{h}=\emptyset$ for $i \neq h$, which is the assumption of Theorem 2.4.

We divide the interval $[1,2]$ into the $n+w+1$ subintervals $\left[1, s_{1}\right]$, $\left[s_{1}, s_{2}\right], \cdots,\left[s_{n+w-1}, s_{n+w}\right],\left[s_{n+w}, 2\right]$, where $s_{v}=1+v /(n+w+1), v=$ $1, \cdots, n+w$. From now on, we construct $\left(\mathcal{B}_{1} \cup \cdots \cup \mathcal{B}_{\mu}\right) \cap R^{3}[1,2]$ so that $\left(\mathcal{B}_{1} \cup \cdots \cup \mathcal{B}_{\mu}\right) \cap R^{3}[0,2]$ forms the required singular-balls. By Theorem 2.4 ; there exist $j \in\{1, \cdots, \mu\}$ and $k \in\{1, \cdots, m(j)+n(j)\}$ so that $S_{j, j}^{(w)}$ is contractible in $R^{3}-\bigcup_{i \neq j} \mathcal{S}_{i}$. Let $g_{1}: D^{3} \rightarrow R^{3}-\bigcup_{i \neq j} \mathcal{S}_{i}$ be a continuous map such that $g_{1}\left(\partial D^{3}\right)=S_{j k}^{(w)}$, and we denote $g_{1}\left(D^{3}\right)$ by $E_{1}$. We set $\mathcal{S}_{j}^{(1)}=\mathcal{S}_{j}-S_{j k}^{(w)}$, and $\mathcal{S}_{i}^{(1)}=\mathcal{S}_{i}$ for $i \neq j$. Then, we define $\left(\mathcal{B}_{1} \cup \cdots \cup \mathcal{B}_{\mu}\right) \cap R^{3}\left[1, s_{2}\right)$ as follows:

$$
\begin{aligned}
& \left(\mathcal{B}_{1} \cup \cdots \cup \mathcal{B}_{\mu}\right) \cap R^{3}[t]=\left(\mathcal{S}_{1} \cup \cdots \cup \mathcal{S}_{\mu}\right)[t] \text { for } 1 \leq t<s_{1}, \\
& \left(\mathcal{B}_{1} \cup \cdots \cup \mathcal{B}_{\mu}\right) \cap R^{3}\left[s_{1}\right]=\left(\mathcal{S}_{1} \cup \cdots \cup \mathcal{S}_{\mu} \cup E_{1}\right)\left[s_{1}\right], \\
& \left(\mathcal{B}_{1} \cup \cdots \cup \mathcal{B}_{\mu}\right) \cap R^{3}[t]=\left(\mathcal{S}_{1}^{(1)} \cup \cdots \cup \mathcal{S}_{\mu}^{(1)}\right)[t] \text { for } s_{1}<t<s_{2} .
\end{aligned}
$$

By Theorem 2.4. there exist $j^{\prime} \in\{1, \cdots, \mu\}$ and $k^{\prime} \in\left\{1, \cdots, m\left(j^{\prime}\right)+\right.$ $\left.n\left(j^{\prime}\right)\right\}$ so that $S_{j^{\prime} k^{\prime}}$ is contractible in $R^{3}-\bigcup_{i \neq j} \mathcal{S}_{i}^{(1)}$. Let $g_{2}: D^{3} \rightarrow$ $R^{3}-\bigcup_{i \neq j} \mathcal{S}_{i}^{(1)}$ be a continuous map with $g_{2}\left(\partial D^{3}\right)=S_{j^{\prime} k^{\prime}}$, and we denote $g_{2}\left(D^{3}\right)$ by $E_{2}$. We set $\mathcal{S}_{j^{\prime}}^{(2)}=\mathcal{S}_{j^{\prime}}^{(1)}-S_{j^{\prime} k^{\prime}}^{(1)}$ and $\mathcal{S}_{i}^{(2)}=\mathcal{S}_{i}^{(1)}$ for $i \neq j^{\prime}$. We define $\left(\mathcal{B}_{1} \cup \cdots \cup \mathcal{B}_{\mu}\right) \cap R^{3}\left[s_{2}, s_{3}\right)$ as follows:

$$
\begin{aligned}
& \left(\mathcal{B}_{1} \cup \cdots \cup \mathcal{B}_{\mu}\right) \cap R^{3}\left[s_{2}\right]=\left(\mathcal{S}_{1}^{(1)} \cup \cdots \cup \mathcal{S}_{\mu}^{(1)} E_{2}\right)\left[s_{2}\right], \\
& \left(\mathcal{B}_{1} \cup \cdots \cup \mathcal{B}_{\mu}\right) \cap R^{3}[t]=\left(\mathcal{S}_{1}^{(2)} \cup \cdots \cup \mathcal{S}_{\mu}^{(2)}\right)[t] \text { for } s_{2}<t<s_{3} .
\end{aligned}
$$

For $R^{3}\left[s_{3}, s_{4}\right), \cdots, R^{3}\left[s_{n+w-1}, s_{n+w}\right), R^{3}\left[s_{n+w}, 2\right)$, we repeat this 
process. It should be noticed that $\mathcal{S}_{1}^{(n+w-1)} \cup \cdots \cup \mathcal{S}_{\mu}^{(n+w-1)}$ consists of a single singular-sphere and $\mathcal{S}_{1}^{(n+w)} \cup \cdots \cup \mathcal{S}_{\mu}^{(n+w)}=\emptyset$. Therefore, $\left(\mathcal{B}_{1} \cup \cdots \cup \mathcal{B}_{\mu}\right) \cap R^{3}\left[s_{n+w}\right]$ consists of a singular-ball $E_{n+w}\left[s_{n+w}\right]$, and $\left(\mathcal{B}_{1} \cup \cdots \cup \mathcal{B}_{\mu}\right) \cap R^{3}[t]=\emptyset$ for $s_{n+w}<t<2$.

Thus, we obtain a union of singular-balls $\mathcal{B}_{i}=B_{i 1} \cup \cdots \cup B_{i n(i)}$ in $R^{3}[0, \infty)$ for $i=1, \cdots, \mu$ such that $\partial^{*} B_{i j}=\Sigma_{i j}$. From our construction, it is easily checked that $\mathcal{B}_{i} \cap \mathcal{B}_{h}=\emptyset$ for $i \neq h$, and this completes the proof of Theorem 4.1.

The relation of link-homotopy was introduced in classical link theory by Milnor $[\mathrm{M}]$, and studied higher dimensional links by MasseyRolfsen $[\mathrm{MR}]$ and Koschorke [K], etc. We record a corollary to Theorem 4.1 on link-homotopy.

4.2. Definition. Let $P_{1}, \cdots, P_{\mu}$ be polyhedra, and let $\mathcal{P}=P_{1} \mathrm{U}$ $\cdots \amalg P_{\mu}$ be their disjoint union, and let $X$ be a manifold. $A$ continuous map $f: \mathcal{P} \rightarrow X$ is said to be a link-map, iff $f\left(P_{i}\right) \cap f\left(P_{h}\right)=\emptyset$ for $i \neq h$. Two link-maps $f_{0}$ and $f_{1}$ of $\mathcal{P}$ into $X$ will be called link-homotopic, iff there exists a homotopy $\left\{\eta_{t}\right\}_{t \in I}: \mathcal{P} \rightarrow X$ such that $\eta_{0}=f_{0}, \eta_{1}=f_{1}$, and $\eta_{t}\left(P_{i}\right) \cap \eta_{t}\left(P_{h}\right)=\emptyset$ for $i \neq h$ and each $t \in I=[0,1]$.

4.3. Theorem. Let $\mathcal{O}_{i}=O_{i 1} \cup \cdots \cup O_{i n(i)}$ be a trivial link in the 3space $R^{3}=R^{3}[0] \subset R^{3}[0, \infty)$ (or $S^{3} \subset \partial D^{4}$ ) for $i=1, \cdots, \mu$, such that $\mathcal{O}_{1} \cup \cdots \cup \mathcal{O}_{\mu}$ is also a trivial link. Let $P_{i}=D_{i 1}^{2} \amalg \cdots \amalg D_{i n(i)}^{2}$ be the disjoint union of $n(i)$ 2-cells for $i=1, \cdots, \mu$, and we set $\mathcal{P}=P_{1} \amalg \cdots \amalg P_{\mu}$. Let $f$ and $e$ be non-degenerate link-maps of $\mathcal{P}$ into $R^{3}$ (or $S^{3}$ ) such that $f\left(\partial D_{i j}^{2}\right)=O_{i j}=e\left(\partial D_{i j}^{2}\right)$ for $i=1, \cdots, \mu$ and $j=1, \cdots, n(i)$.

Then, $f$ and e are link-homotopic in $R^{3}\left[0, \infty\right.$ ) (or $D^{4}$ ) keeping $\mathcal{O}_{1} \cup$ $\cdots \cup \mathcal{O}_{\mu}$ fixed.

Proof. Let $f\left(D_{i j}^{2}\right)=D_{i j}$ and $\mathcal{D}_{i}=D_{i 1} \cup \cdots \cup D_{i n(i)}$ for $i=1, \cdots, \mu$ and $j=1, \cdots, n(i)$. Let $g: \mathcal{P} \rightarrow R^{3}$ be an embedding, and let $g\left(D_{i j}^{2}\right)=$ $D_{i j}^{*}$ and $\mathcal{D}_{i}^{*}=D_{i 1}^{*} \cup \cdots \cup D_{i n(i)}^{*}$. In this notation, it suffices to show that $f$ and $g$ are link-homotopic in $R^{3}[0, \infty)$ keeping $\mathcal{O}_{1} \cup \cdots \cup \mathcal{O}_{\mu}$.

In the notation of Theorem 4.1, we have a finite union of singularballs $\mathcal{B}_{1} \cup \cdots \cup \mathcal{B}_{\mu}, \mathcal{B}_{i}=B_{i 1} \cup \cdots \cup B_{i n(i)}$ in $R^{3}[0, \infty)$ such that $B_{i} \cap \mathcal{B}_{h}=\emptyset$ for $i \neq h$ and $\partial^{*} B_{i j}=\Sigma_{i j}$. Let $b_{i j}: D^{2} \times I \rightarrow R^{3}[0, \infty)$ be a continuous 
map of the 3-cell $D^{2} \times I$ such that $b_{i j}\left(D^{2} \times I\right)=B_{i j}$. We may assume that $b_{i j}\left|D^{2} \times 0=f\right| D_{i j}^{2}$ and $b_{i j}\left|D^{2} \times 1=g\right| D_{i j}^{2}$. Then, associating with these $b_{i j}$, we have a link-homotopy $\left\{\eta_{t}\right\}_{t \in I}: \mathcal{P} \rightarrow R^{3}[0, \infty)$ defined by

$$
\eta_{t}\left(D_{i j}^{2}\right)=b_{i j}\left(D^{2} \times t\right)
$$

for every $t \in I$. From the condition of the singular-balls $B_{1} \cup \cdots \cup B_{\mu}$ in Theorem 4.1 , it is easily checked that this homotopy $\left\{\eta_{t}\right\}_{t \in I}$ between $f$ and $g$ satisfies our required condition, and completing the proof of Theorem 4.3.

\section{References}

[F] Fox, R.H.: On the imbedding of polyhedra in 3-space. Ann. of Math. (2), 49 (1948), 462-470.

[H] Hudson, J.F.P.: Piecewise Linear Topology. W.A. Benjamin, New York, 1969.

[KSS] Kawauchi, A., Shibuya, T. and Suzuki, S.: Descriptions on surfaces in four-space I. Math. Sem. Notes Kobe Univ., 10 (1982), 75-125.

[K] Koschorke, U.: Higher-order homotopy invariants for higher-dimensional link maps. Lecture Notes in Math., 1172 (1985), Springer-Verlag, 116-129.

[MR] Massey, W.S. and Rolfsen, D.: Homotopy classification of higher dimensional links. Indiana Univ. Math. J., 34 (1985), 375-391.

[M] Milnor, J.: Link groups. Ann. of Math. (2), 59 (1954), 177-195.

[RS] Rourke, C.P. and Sanderson, B.J.: Introduction to Piecewise-Linear Topology. Ergebn. Math. u. ihrer Grenzgeb. Bd. 69, Springer-Verlag, Berlin-Heidelberg-New York, 1972.

[P] Papakyriakopoulos, C.D.: On Dehn's lemma and the asphericity of knots. Ann. of Math. (2), 66 (1957), 1-26.

Department of Mathematics Kobe University

Nada-ku, Kobe 657, Japan
Department of Matbematics Waseda University

Shinjuku-ku, Tokyo 169-50, Japan

Recibido: 25 de Febrero de 1994 